\title{
Annular arrays for novel ultrasonic measurement techniques
}

\author{
Mario Wolf, Elfgard Kühnicke, Sebastian Kümmritz, and Michael Lenz \\ Solid-State Electronics Laboratory, Dresden University of Technology, Dresden, Germany \\ Correspondence to: Mario Wolf (mario.wolf@tu-dresden.de)
}

Received: 29 April 2016 - Revised: 30 August 2016 - Accepted: 22 September 2016 - Published: 1 November 2016

\begin{abstract}
The paper shows how the precise knowledge of the sound field of an ultrasonic annular array can contribute to the development of novel measurement techniques. Emphasis is placed on (a) a non-invasive method for sound velocity measurements in fluids using the echo signals from scattering p]articles, (b) a non-invasive method for the combined determination of thickness and sound velocity in layered structures by using a novel focusing technique, and (c) a non-scanning curvature measurement method exploiting the wave front curvature of a reflected wave. To demonstrate the methods, the principles as well as results of simulations and measurements are discussed.
\end{abstract}

\section{Introduction}

Conventional ultrasonic pulse-echo methods measure the time of flight $(\mathrm{ToF})$ as the only quantity. This is used to determine the sound velocity or the distance of a reflector, while the other quantity has to be known. For imaging in non-destructive testing (NDT) or in medical diagnostics, the value of sound velocity is assumed a priori. Interfaces of hidden objects are imaged by the measured ToF. The supposed locations and dimension of these objects are incorrect if the actual sound velocity differs from the assumed one. For material characterization, the thickness of a specimen is determined by mechanical measurements and the sound velocity is determined by ToF measurements. For multi-layered structures the mechanical or optical determination of the thickness of the different layers is impossible non-destructively. It is necessary to determine both quantities simultaneously to get information about the thickness and the material of the different layers.

To measure sound velocity locally resolved by means of tomography, usually a high number of reflectors or transducers at known positions are required (Huang et al., 2004) and the test object has to be accessible from all directions. A method for the simultaneous measurement of thickness and sound velocity for a single-layer specimen with coplanar surfaces is introduced by Hsu et al. (1992) and Dayal (1992) and pursued by Kim et al. (2003). For that method, the specimen has to be placed in a medium with a known sound velocity and between two probes with a known distance. The ToFs of different reflected and transmitted signals are evaluated to determine the thickness and sound velocity of the plate. Such an invasive method is suitable for small and not embedded components and is qualified only for an one-layered specimen. Loosvelt and Lasaygues (2011) use this method for plates with a thickness smaller than the wavelength.

This paper demonstrates how additional information of the reflected sound field is gathered by using segmented annular arrays. New measurement techniques have been developed, being characterized by a close combination of sound field simulations and measurements. They use synthetic or electronic focusing and analyse the phases and amplitudes on all transducer elements to determine the object curvature radius or sound velocity and distance simultaneously. These approaches require the precise knowledge of the sound field dependent on the array parameters and the set-up. Therefore, sound field simulations are necessary.

A half-analytical method has been developed to optimize transducers and to calculate the sound field in complex structures and layered media. It is based on time-harmonic Green's functions in a steepest descent approximation for a two-dimensional geometry. The source area is discretized and uniformly covered with point sources. The sound field that is irradiated from a finitely extended source is the superposition of the field of all point sources. The sound field in 

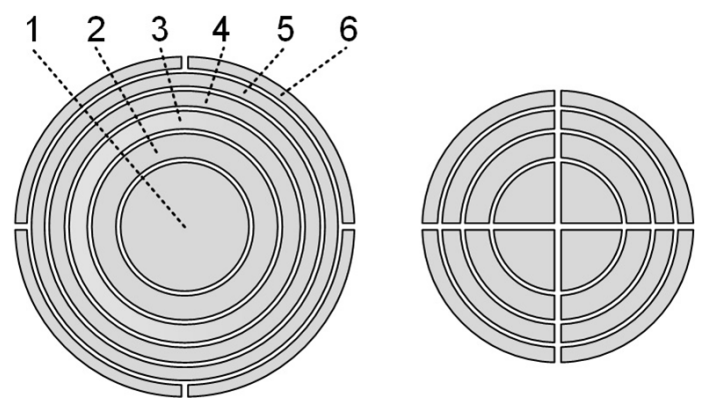

Figure 1. Geometry of the annular arrays: the numbering used in the following paragraphs starts at one for the central element and ends with six for the fifth ring; e.g. $s_{16}$ is the signal emitted from the central element and received at the fifth ring.

a layered medium can be calculated approximately by a decomposition of the medium into different layers and a separate calculation of wave propagation in each layer (Kühnicke, 2007). This method is applied for each element to simulate the field generated in the medium or at the different interfaces, including the reflections back to the transducer. The applied focusing techniques can be realized by phase shifting and superposing the different fields.

Different annular arrays are used (Fig. 1). They have six active ring elements and, depending on the task, a curved interface for a small focus extension or additional segments for non-axis-symmetric problems or adjustment (Fig. 1, right). The centre frequencies are between 3 and $10 \mathrm{MHz}$, and the central elements have diameters from 4 to $5 \mathrm{~mm}$ with results in near-field lengths between 10 and $30 \mathrm{~mm}$. The outer rings have the same area and so the same near-field length, which enables a well focusing. The active material is a composite made by the dice and fill technique (Janas and Safari, 1995).

\section{Scattering particle method for locally resolved measurement of sound velocity}

The monitoring of sound velocity is of great interest in chemical processes because sound velocity is a measure for concentration, mass density and elastic parameters. Other possible applications of locally resolved sound velocity measurements are tissue characterization and noninvasive temperature monitoring during hyperthermia for cancer therapy.

Conventional pulse-echo methods measure the ToF to determine only one quantity: the sound velocity or the distance of a reflector. However, the other quantity has to be known. To measure a local distribution of sound velocity, usually further reflectors or transducers at known positions are required. These additional mechanical parts cause a high instrumentation effort. If the examined medium is not all-round accessible, transducers/reflectors have to be inserted, which can affect the examined process. Therefore, a non-invasive method
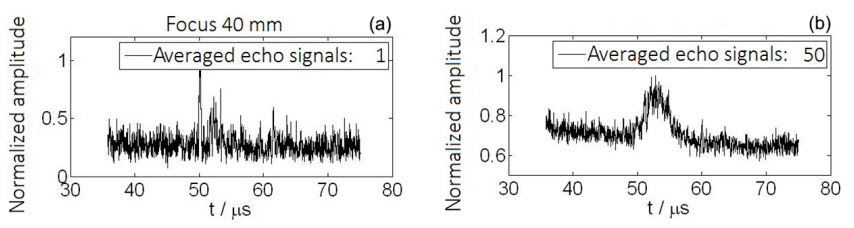

Figure 2. Normalized amplitude of averaged echo signals of an annular array at focusing $F o k=40 \mathrm{~mm}$ in water of $30^{\circ} \mathrm{C}$ $\left(c=1510 \mathrm{~m} \mathrm{~s}^{-1}\right)$ for different numbers of averages (Maximum at $t=52 \mu \mathrm{s})$.
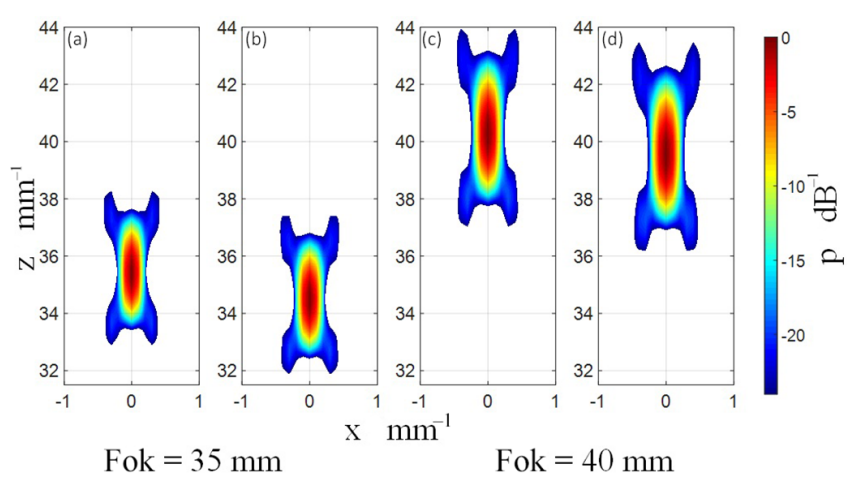

Figure 3. Calculated sound fields (focusing in send and receive mode) dependent on the sound velocity in the propagation medium for a curved $9 \mathrm{MHz}$ annular array at different control modes Fok.

without the need for fixed reflectors is most desirable to measure sound velocity profiles.

This paper presents a novel approach for simultaneous measurement of sound velocities and distances in fluids containing moving scattering particles. Because the sound field of an ultrasonic transducer depends on both the transducer parameters and the sound velocity of the propagation medium, the focus position is used as a second measuring quantity instead of using only the propagation time. The focus position is determined by evaluating the echoes of the scatterers. The echo of a single particle becomes strongest if it is located in the focal zone of the sound field. Because particles are in motion in a fluid, it is possible to assume a uniform distribution of particles in time average. So the averaged amplitude of echo signals becomes maximal for the ToF to the focus and back (Fig. 2).

Lenz et al. (2011) and Lenz and Kühnicke (2012) give proof of concept by measurements in fluids covering a wide range of sound velocities with a strongly focusing transducer equipped with a lens. An uncertainty in velocity determination of less than $0.1 \%$ was achieved in a detailed measurement using water at different temperatures.

To achieve a local resolution of the sound velocity measurement along the axis without additional mechanical effort, an ultrasonic array is required. Due to the available electronic instrumentation, first measurements were done by emission with one element and synthetic focusing of the received sig- 

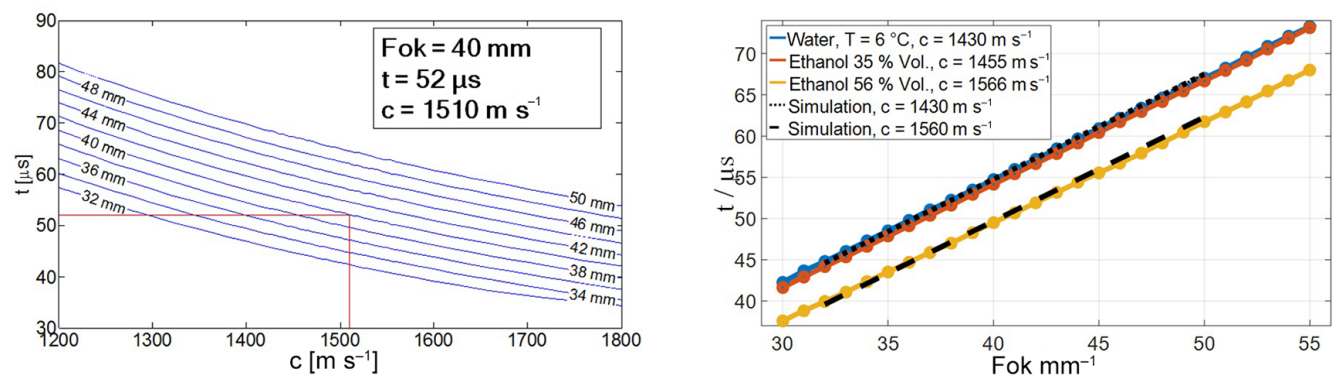

Figure 4. Left: calibration curve - time of flight as a function of sound velocity for different control modes; right: time of flight as a function of control mode for different sound velocities.

nals, which means that signals are time shifted and superposed (Kühnicke et al., 2011). The development of a new pulser allows driving all elements together, whereby each pulse can be delayed with a definite time lag. For a second focusing, the received signals are superposed and thus focused synthetically. This reduces the statistical uncertainty because it minimizes the focus extension and contributes to a larger signal-to-noise ratio.

The control mode Fok corresponds to a set of delay times. The delay times are chosen in such a way that the focus of the transducer is located at the distance $z=F o k$ in the calibration medium water at room temperature. This set of delay times is used for all measurements, independently from the real sound velocity.

Because the focus position depends on the sound velocity of the medium, sound field simulations are required to predict the focus position as a function of the sound velocity of the medium, where the parameters of the array need to be considered, as well. Figure 3 shows calculated sound fields of the used curved annular array for two different control modes and for two different sound velocities of the propagation medium.

Figure 3 confirms that the focus position depends on the velocity of the propagation medium (compare Fig. 3a with b, and $\mathrm{c}$ with $\mathrm{d}$ ). The position of the sound field maximum can be varied by means of control mode (compare Fig. 3a with c, and $b$ with $d)$.

To create calibration curves, the sound fields are simulated for different sound velocities (and different control modes). The positions of the sound field maxima are then converted into the corresponding ToFs. Plotting the ToFs as a function of sound velocity for several control modes Fok leads to the series of calibration curves depicted in Fig. 4. Using the measured ToF in an unknown fluid and considering the used control mode Fok then enables one to read off the sound velocity of the medium using Fig. 4, left.

To compare measurements and simulations, echoes of scattering particles and therefore sound velocities and focus positions are measured in media with constant temperature (Fig. 4, right). It is striking that the measured curves fit very well to the simulations.
Table 1. Reachable uncertainty.

\begin{tabular}{lll}
\hline $\begin{array}{l}\text { Number of } \\
\text { averaged signals }\end{array}$ & $\begin{array}{l}\text { Standard deviation } \pm 3 \sigma \\
\text { of time of flight }\end{array}$ & $\begin{array}{l}\text { Absolute error } \\
\text { of sound velocity }\end{array}$ \\
\hline 50 & $500 \mathrm{~ns}$ & $13 \mathrm{~m} \mathrm{~s}^{-1}$ \\
200 & $250 \mathrm{~ns}$ & $6 \mathrm{~m} \mathrm{~s}^{-1}$ \\
1000 & $150 \mathrm{~ns}$ & $4 \mathrm{~m} \mathrm{~s}^{-1}$ \\
5000 & $100 \mathrm{~ns}$ & $2.5 \mathrm{~m} \mathrm{~s}^{-1}$ \\
\hline
\end{tabular}
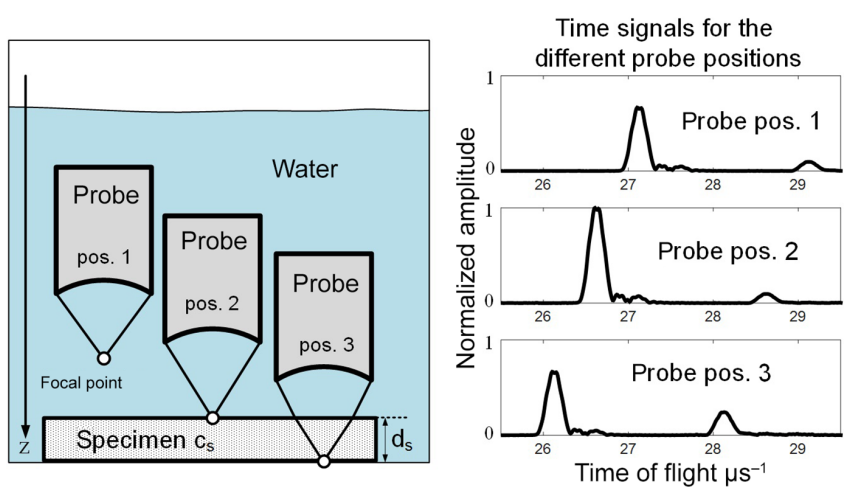

Figure 5. Principle drawing for the combined measurement of thickness and sound velocity by means of a focusing transducer.

The method has been qualified for media with constant sound velocity, and for these measurements, an accuracy of more than $99.8 \%$ was reached (Wolf et al., 2013). It had been shown that the measurement uncertainty for constant sound velocities can be reduced to $2.5 \mathrm{~m} \mathrm{~s}^{-1}$, which is less than $2 \%$, in water (see Table 1).

The current work deals with generating calibration curves for media with continuously changing properties. The continuous change of material properties is taken into account by a modified wave equation. The potential of a point source has been calculated in the Hankel transformed domain. The inverse transform is realized and allows calculating Green's functions for media with continuously changing properties for a change of material properties in axial direction of the array (Wolf and Kühnicke, 2015). A change of these proper- 

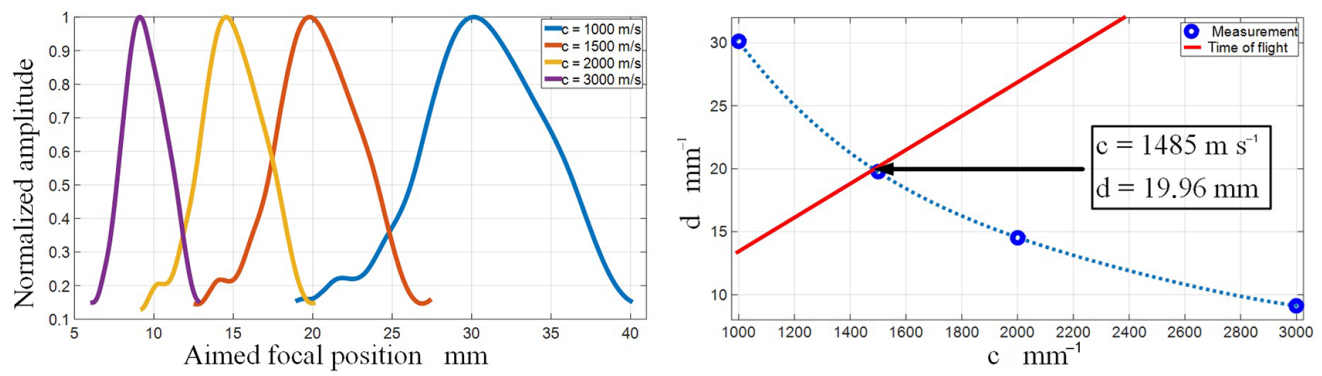

Figure 6. Left: focusing curves for different control sound velocities; right: determination of sound velocity by intersection of two curves; Red curve: reflector distance as a function of sound velocity and measured ToF. Blue curve: reflector distance as a function of the control sound velocity $c_{\text {control }}$; Blue points: four pairs of sound velocity and corresponding distance are determined by focusing curves.

ties in other directions would cause a lateral deviation of the focus position and has to be examined in further works. Further advances of the method are planned for measuring sound velocity profiles in media with stationary particles (e.g. medical tissues).

\section{Simultaneous determination of thickness and sound velocity}

For imaging in NDT or in medical diagnostics, the value of sound velocity is assumed a priori. Interfaces of hidden objects (defects, surfaces of organs, vessels) are imaged by the measured ToF. The supposed locations and the extensions of objects are incorrect if the real speed of sound differs from the assumption.

For material characterization the thickness of a specimen is measured (e.g. mechanically) and the sound velocity is determined by ToF measurements. If there is a multi-layered structure, the mechanical determination of the thickness of the different layers can impossibly be done non-destructively. It is necessary to determine both quantities simultaneously to get information about the thicknesses and the materials of the different layers.

To satisfy the demands of NDT, novel approaches evaluate the sound field, which depends on the propagation medium. Gust (2011) determines sound velocity and thickness of plates with a scanning acoustic microscope by moving a probe in water vertically to the surface. The focus position is used as a second measuring quantity in addition to the propagation time. The echo of the surface and of the back wall is maximal if the focus is located on it (compare signals for position 2 and 3, Fig. 5). The displacement between the transducer positions, where the echo of the first and the second interface become strongest, is determined. Because the focus position depends on the sound velocity, the necessary displacement differs dependent on the material of the plates, even for plates of the same thickness. So the displacement and the ToF between the interfaces can be used to determine sound velocity and thickness simultaneously.
A method is introduced by Kümmritz et al. (2015) allowing a simultaneous determination of sound velocity and thickness of up to two layers with an annular array at a fixed position. It works by varying the control mode to move the focus position along the acoustic axis. The amplitudes of the echo signals are evaluated to get additional information beside the ToF. A measurement set-up with a water delay line between the specimen and a plane annular array with a centre frequency of $6 \mathrm{MHz}$ is used.

The approach is explained by analysing a first liquid layer. The focus position is continuously moved along the acoustic axis by changing the control mode calculated on the basis of different assumed control sound velocities $c_{\text {control }}$. In contrast to the approach with scatterers the control sound velocity is varied. In Fig. 6 (right curve), a control sound velocity of $1000 \mathrm{~m} \mathrm{~s}^{-1}$ is used to calculate the delay times. The back wall echo signal is determined, and its amplitude is recorded as a function of the control mode. The result is the blue focusing curve on the right side. At $30 \mathrm{~mm}$, a maximum can be seen. This maximum indicates that if the sound velocity of the fluid was $1000 \mathrm{~m} \mathrm{~s}^{-1}$, the thickness of the first layer would be $30 \mathrm{~mm}$. The process is repeated for different control velocities $c_{\text {control }}$ (Fig. 6, left), and the supposed thickness is recorded as a function of control sound velocity (Fig. 6, right blue line).

The ToF between probe and surface gives additional information (red line). The correct sound velocity and thickness of the specimen is the intersection of the two curves.

After the determination of thickness and velocity of the first layer, the approach is repeated for the second layer. Refraction effects have to be taken into account to correctly focus into the second medium. The delay times are determined by means of Fermat's principle. The thickness and sound velocity of the first layer have to be known for this.

Measurements were done for a two-layered structure with the first layer of water and the second layer of steel or aluminum with a thickness $d=6,8,10,12$ and $14 \mathrm{~mm}$ (Kümmritz et al., 2015). The relative error was less than $1 \%$. For the second layer, a relative error of less than $3 \%$ had been achieved for the current measurement set-up. 

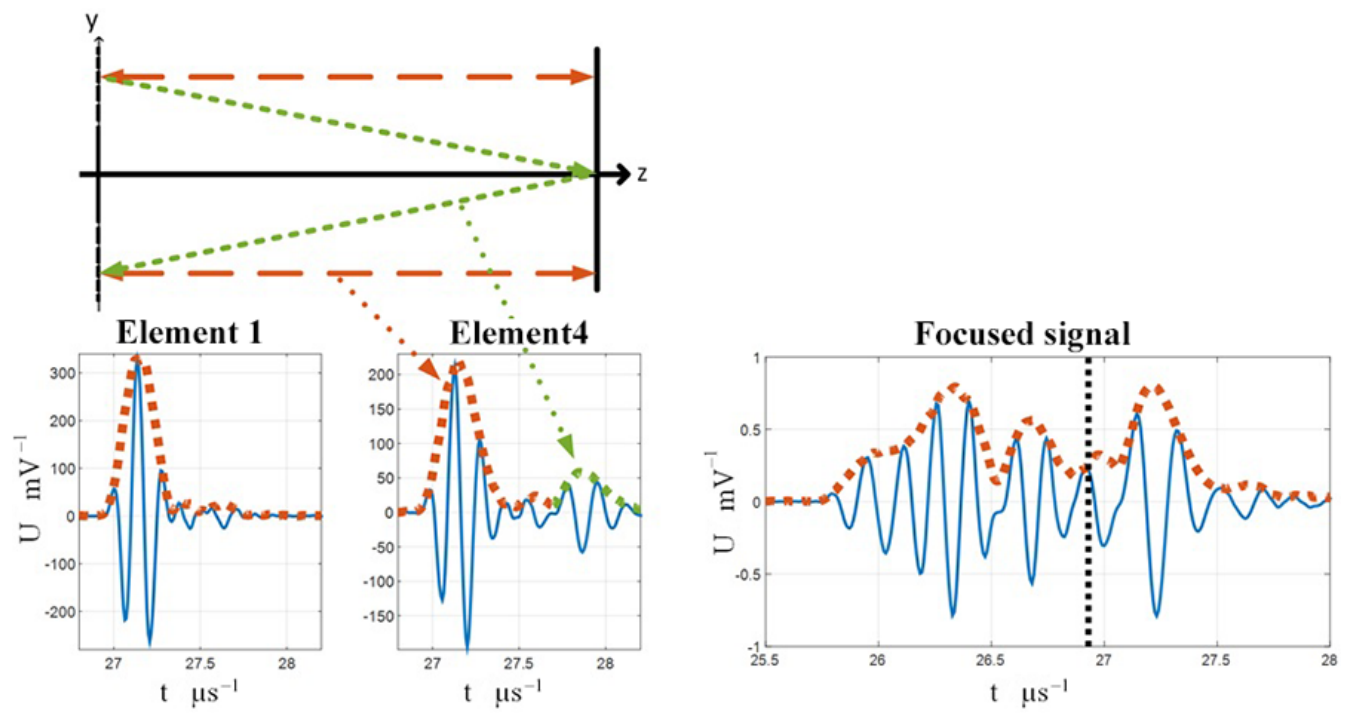

Figure 7. Signals for different propagation paths.

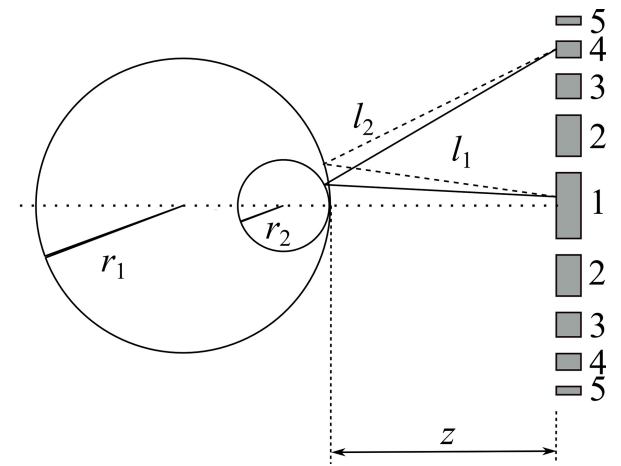

Figure 8. The time of flight of the ultrasonic wave between the inner and outer transducer elements (numbers 1-5) of an annular array depends on the reflector curvature. The larger the sphere, the shorter the propagation path is from the innermost element to the outer elements.

With this approach the delay times, where the focused echo signal becomes maximal, are iteratively determined, which means that they interfere maximally positively. Figure 9 exemplarily shows the signals transceiving with the central element and the third ring. Whereas there is only one signal for the central element, two signal parts can be recognized for the third ring. The two signal parts can be related geometrically to two different propagation paths: the direct wave with normal incidence and the wave which is received at the opposite site of the ring (see Fig. 7, left). The second signal part of the third ring has to interfere with the single signal of the central element for optimal focusing. This means that the "correct" delay times can be determined directly from the signal without focusing, which leads to another ap- proach determining thickness and sound velocity with these delay times and an inverse model.

As a first step the signal parts have to be separated as they interfere for the inner elements. The signal of the central element is chosen as reference signal because it has a propagation path with normal incidence. Due to small phase shifts in the signal first the analytic signal $s_{i j}^{\mathrm{H}}$ is calculated by Hilbert transform of signal $s_{i j}$ emitted by element $i$ and received by element $j$.

The mean phase difference is calculated and used to shift the reference signal. Finally the real part of the difference of analysed and phase shifted reference signal is calculated. This yields a difference signal $s^{\mathrm{D}}$ containing only the second signal part.

$s_{j i}^{\mathrm{D}}(t)=\Re\left[s_{i j}^{\mathrm{H}}(t)-s_{11}^{\mathrm{H}}(t) e^{j\left(\left(s_{j i}^{\mathrm{H}}(t)\right)-\left(s_{11}^{\mathrm{H}}(t)\right)\right)}\right]$

The "correct" delay time is now determined by crosscorrelating $s_{i j}^{\mathrm{D}}$ and $s_{11}$.

Additionally a geometric model is applied. It calculates the ToF as a function of thickness and sound velocity of the second layer.

$t=2 \frac{\sqrt{\left(y_{\mathrm{E}}-y_{\mathrm{D}}\right)^{2}-z_{1}^{2}}}{c_{1}}+2 \frac{\sqrt{\left(y_{\mathrm{D}}-y_{\mathrm{R}}\right)^{2}+z_{2}^{2}}}{c_{2}}$,

with

$y_{\mathrm{E}}=r_{i}, y_{\mathrm{R}}=\left(r_{i}+r_{j}\right) / 2$,

where $r_{i}$ and $r_{j}$ are the mean radii of emitter $i$ and receiver $j, z_{1}$ and $c_{1}$ the (known) thickness and sound velocity of the first medium, $z_{2}$ and $c_{2}$ the (unknown) thickness and sound velocity of the investigated layer. Also the coordinate $y_{\mathrm{D}}$ of 

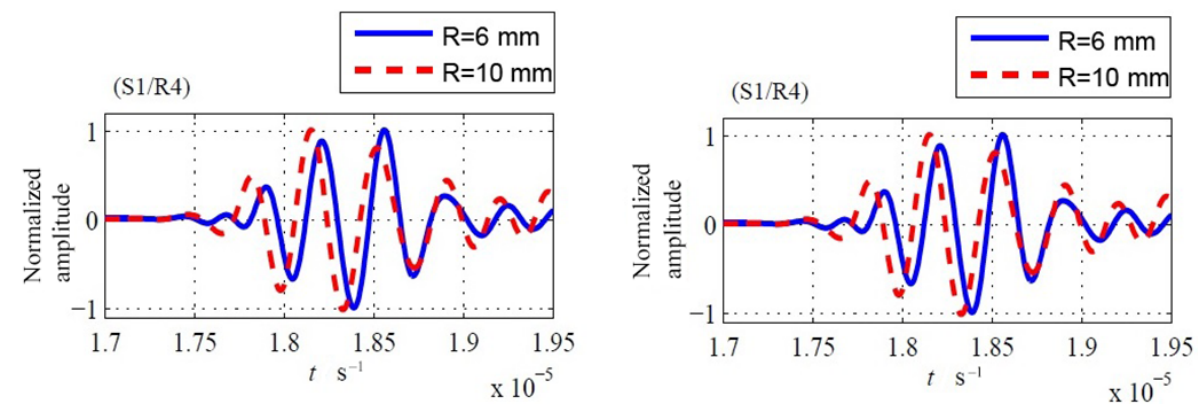

Figure 9. Measured echo signals for the propagation paths (S1/R1) and (S1/R4) of a plane annular array with five elements for sphere radii of 6 and $10 \mathrm{~mm}$ (S: sending element, R: receiving element). The sphere is located at $z=13 \mathrm{~mm}$, which is the near-field length of the single elements of the annular array.

Table 2. Comparison of time differences between the two echo parts gained from sound field simulation $\left(t_{\text {sim }}\right)$ and determined with a geometric model via Fermat's principle $\left(t_{\mathrm{geo}}\right)$.

\begin{tabular}{rrrrrr}
\hline & $t_{22}[\mathrm{~ns}]$ & $t_{33}[\mathrm{~ns}]$ & $t_{44}[\mathrm{~ns}]$ & $t_{55}[\mathrm{~ns}]$ & $t_{66}[\mathrm{~ns}]$ \\
\hline$t_{\text {sim }}$ & 160 & 212 & 276 & 396 & 540 \\
$t_{\text {geo }}$ & 97 & 197 & 299 & 405 & 548 \\
\hline
\end{tabular}

the point $P_{\mathrm{D}}$ is unknown, but it can be determined by Fermat's principle, meaning that the ToF must be minimal. Taking into account that $z_{2}$ is connected with $c_{2}$ by the ToF $T$ between surface and back wall echoes it can be replaced with $z_{2}=c_{2} T_{2} / 2$. This leads to

$$
\begin{array}{r}
t_{i j}=f\left(c_{2}\right)=2 \frac{\sqrt{\left[y_{E, i j}-y_{\mathrm{D}}\left(c_{2}\right)\right]^{2}-z_{1}^{2}}}{c_{1}} \\
+2 \frac{\sqrt{\left[y_{\mathrm{D}}\left(c_{2}\right)-y_{\mathrm{R}, i j}\right]^{2}+\left(c_{2} T / 2\right)^{2}}}{c_{2}},
\end{array}
$$

where $t_{i j}$ is a function of the (solely unknown) variable $c_{2}$. Combining the measured time differences $\Delta t_{i j}$ with these functions allows the determination of $c_{2}$ with the leastsquares method.

$F=\sum_{i=1}^{N} \sum_{j=1}^{N}\left(\Delta t_{i j}-\left(t_{i j}\left(c_{2}\right)-t_{11}\left(c_{2}\right)\right)\right)^{2} \rightarrow \min$

Of course, the determined sound velocity provides the thickness of the layer.

As this geometric model neglects the wave behaviour of the sound, additional sound field simulations are done. The reflected signal is calculated by a harmonic synthesis convolving the excitation signal with the frequency components of the sound field. As the thickness and sound velocity are defined in simulation these calculated signals can be used to compare the resulting time differences with them from the geometric model. An example is shown in Table 2.
Table 3. Different attaching points for geometric model used in Eq. (4): $y_{\mathrm{E}, \text { geo }}$ gained from main radii of array electrodes; $y_{\mathrm{E}, \mathrm{corr}}$ adapted points so that time differences (see Table 3) from geometric model and from sound field simulations agree.

\begin{tabular}{lrrrrrr}
\hline Element & 1 & 2 & 3 & 4 & 5 & 6 \\
\hline$y_{\mathrm{E}, \text { geo }}[\mathrm{mm}]$ & 1.58 & 2.90 & 3.85 & 4.64 & 5.34 & 6.19 \\
$y_{\mathrm{E}, \text { corr }}[\mathrm{mm}]$ & 1.58 & 3.24 & 3.55 & 4.76 & 5.47 & 6.39 \\
\hline
\end{tabular}

As expected the times differ significantly because the geometric model would only be valid for plane waves. The coordinate $y_{\mathrm{E}}$ in the geometric model had been chosen as the mean radius of the element $\left(y_{\mathrm{E}, \mathrm{geo}}\right)$ but can be chosen arbitrarily. So the sound field simulations can be used to modify the model parameters: $y_{\mathrm{E}, \mathrm{corr}}$ is determined so that the geometric model gives the times gained from simulation. Table 3 compares the mean radii $y_{\mathrm{E}, \text { geo }}$ with the corrected ones $y_{\mathrm{E} \text {,corr }}$ and Table 4 the sound velocities determined with both.

\section{Curvature measurements}

When measuring in pulse-echo mode, the reflected ultrasonic wave contains not only information about material parameters such as the sound velocities in a layered structure but also some information about the local inclination angle toward the acoustic axis and the curvature of the reflector.

While the information about the reflector inclination is mainly contained in the angle of reflection of the reflected wave, the information about the reflector curvature is contained in the wave front curvature and the arrival times of the reflected wave on the different elements of an annular array.

In Fig. 8 it can be seen that the propagation path lengths between the inner element of an annular array and the outer elements show a dependence on the reflector radius.

In Fig. 9 measurements were done to distinguish reflectors and determine the reflector curvature of spheres being kept in a water bath. While the ToF of the wave propagating along the acoustic axis is the same for both reflector radii 
Table 4. Comparison of determined sound velocities with the attaching points $y_{\mathrm{E}, \text { geo }}$ (from array geometry) and corrected $y_{\mathrm{E}, \text { corr }}$ via sound field simulations.

\begin{tabular}{lrrrrr|rrrrr}
\hline Delay line & \multicolumn{4}{c|}{$15 \mathrm{~mm}$} & \multicolumn{5}{c}{$20 \mathrm{~mm}$} \\
\hline$t d[\mathrm{~mm}]$ & 4 & 6 & 8 & 10 & 14 & $4 \mathrm{~mm}$ & $6 \mathrm{~mm}$ & $8 \mathrm{~mm}$ & 10 & 14 \\
$c_{\text {geo }}\left[\mathrm{m} \mathrm{s}^{-1}\right]$ & 5688 & 5787 & - & - & 5649 & 5682 & 5765 & 5647 & 5772 & 5688 \\
$c_{\text {corr }}\left[\mathrm{m} \mathrm{s}^{-1}\right]$ & 5866 & 5920 & 5923 & 5960 & 5929 & 5883 & 5902 & 5909 & 5939 & 5866 \\
\hline
\end{tabular}
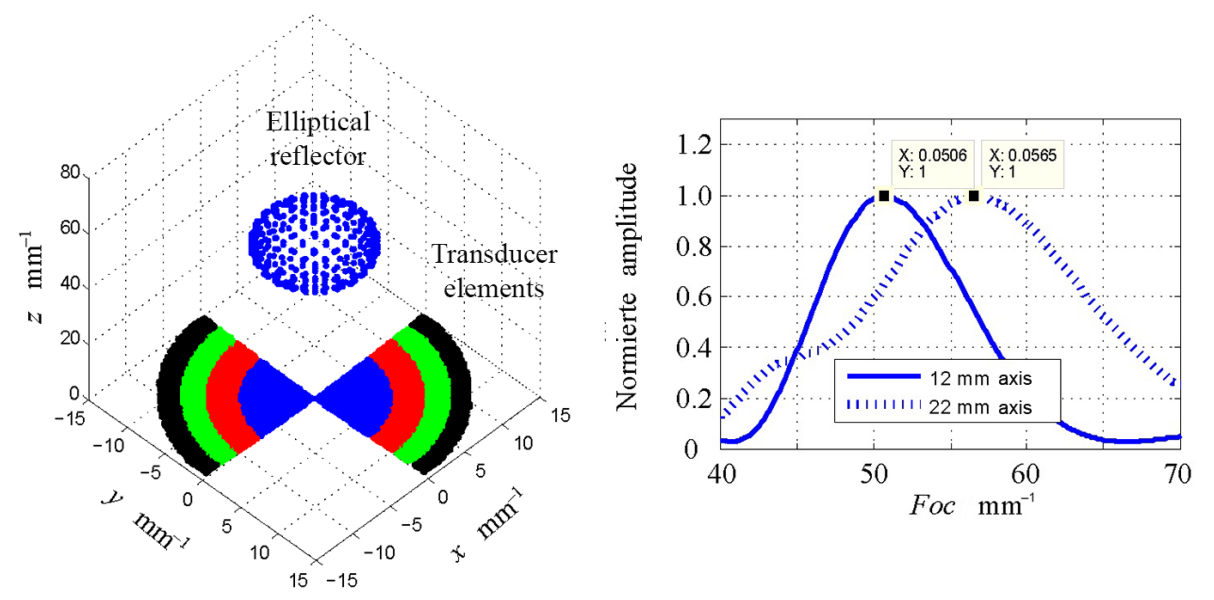

Figure 10. Focused sound emission with two opposite $90^{\circ}$ transducer segments each being divided in radial direction into four elements (left figure). The focusing curves allow the determination of the half-axes of the reflector (right figure).

(Fig. 9, left), the ToF from the innermost element to the outer element with number four depends on the reflector radius (Fig. 9, right). Under the given conditions, sphere radii could be determined with an uncertainty of about $0.5 \mathrm{~mm}$, where spheres of between 6 and $11 \mathrm{~mm}$ radius were used in the experiments (Lenz, 2013).

A further segmentation of the annular array in four $90^{\circ}$ pieces enables the differentiation of the half-axes of a rotational ellipsoid. In the following measurements, the $90^{\circ} \mathrm{seg}-$ ments on the opposite sides of the transducer were always used together, where the half-axes of the reflector were put parallel to the symmetry axes of the transducer (Fig. 10, left).

Instead of measuring time delays of different propagation paths, as in the set-up of Fig. 9, the transducer was focused here and the echo signal amplitude was plotted as a function of the focus setting Fok, i. e. the set of delay times focusing at a distance of $z=F o k$ on the acoustic axis. As can be seen in Fig. 10 (right), the different half-axes of an elliptical reflector can be distinguished by comparing the maximum positions of the focusing curves.

While the main drawback of the presented non-scanning curvature measurement methods is the necessity for large transducer apertures and the high cross-sensitivity of the method towards local inaccuracies of the surface, the methods might be useful in the future to achieve additional pieces of information in scanning and non-scanning applications in NDT and medical engineering.

\section{Data availability}

The data are not available online. Please contact the authors to obtain access.

\section{Summary}

Three approaches have been introduced which use the wave properties of ultrasound instead of measuring simply the ToF. They allow the measurement of the sound velocity in fluids and solids with one transducer and without the need of additional reflectors at known positions. Whereas the scattering particle method shall be applied for process monitoring and medical diagnostics the method for measuring curvature, thickness and sound velocity shall be applied in NDT and for material characterization. The development of all methods required sound field simulations to predict the pressure distribution on the transducer and to find evaluation criteria.

Acknowledgements. The authors would like to thank Deutsche Forschungsgemeinschaft (DFG) for their financial support of the ongoing research project KU1075/17-1.

Edited by: O. Kanoun

Reviewed by: three anonymous referees 


\section{References}

Dayal, V.: An automated simultaneous measurement of thickness and wave velocity by ultrasound, Experimental mechanics, 197202, September 1992.

Gust, N.: Improvement of signal analysis for ultrasonic microscopy, Dissertation Technische Universität Dresden, ISBN-13: 978-3942710-22-0, 2011.

Huang, S.-H. and Li, P.-C.: Computed Tomography Sound Velocity Reconstruction Using Incomplete Data, IEEE T. Ultrason. Ferr., 51, 329-342, 2004.

Hsu, D. K. and Hughes, M. S.: Simultaneous ultrasonic velocity and sample thickness measurement and application in composites, J. Acoust. Soc. Am., 92, 669-675, 1992.

Janas, V. and Safari, A.: Overview of Fine-Scale Piezoelectric Ceramic/Polymer Composite Processing, J. Am. Ceram. Soc., 78, 2945-2955, doi:10.1111/j.1151-2916.1995.tb09068.x, 1995.

Kim, Y. H., Song, S., and Lee, J.: Simultaneous measurements of the ultrasonic wave velocity and thickness of a solid plate made from one side of the plate, Measurement Science and Technology, 14, p. N13, 2003.

Kühnicke, E.: Plane arrays - Fundamental investigations for correct steering by means of sound field calculations, Wave Motion, 44, 248-261, doi:10.1016/j.wavemoti.2006.10.003, 2007.

Kühnicke, E., Lenz, M., and Bock, M.: Non-invasive measurement of sound velocity profiles, IEEE International Ultrasonics Symposium, 2151-2153, Orlando, USA, 18-21 October 2011.
Kümmritz, S., Wolf, M., and Kühnicke, E.: Simultane Bestimmung von Dicken und Schallgeschwindigkeiten geschichteter Strukturen, tm - Technisches Messen 82/3, 127-134, 2015.

Lenz, M., Bock, M., Kühnicke, E., Pal, J., and Cramer, A.: Measurement of the sound velocity in fluids using the echo signals from scattering particles, Ultrasonics, 52.1, 117-124, doi:10.1016/j.ultras.2011.07.003, 2011.

Lenz, M. and Kühnicke, E.: Nichtinvasive Schallgeschwindigkeitsmessung in Fluiden auf Basis von Streupartikelechos, tm - Technisches Messen 79/1, 23-28, doi:10.1524/teme.2012.0175, 2012.

Lenz, M.: Neuartige Ultraschallmeßverfahren unter Nutzung von Schallfeldinformationen, Dissertation Technische Universität Dresden, available at: http://nbn-resolving.de/urn:nbn:de:bsz: 14-qucosa-135656 (last access: 18 October 2016), 2013.

Loosvelt, M. and Lasaygues, P.: A Wavelet-Based Processing method for simultaneously determining ultrasonic velocity and material thickness, Ultrasonics, 51, 325-339, 2011.

Wolf, M., Kühnicke, E., and Lenz, M.: Modeling of sound propagation in media with continuously changing properties towards a locally resolved measurement of sound velocity, IEEE International Ultrasonics Symposium (IUS), 1045-1048, Prague, Czech Republic, 21-25 July 2013.

Wolf, M. and Kühnicke, E.: Non-invasive and Locally Resolved Measurement of Sound Velocity by Ultrasound, Sensors \& Transducers, 184/1, 53-59, 2015. 